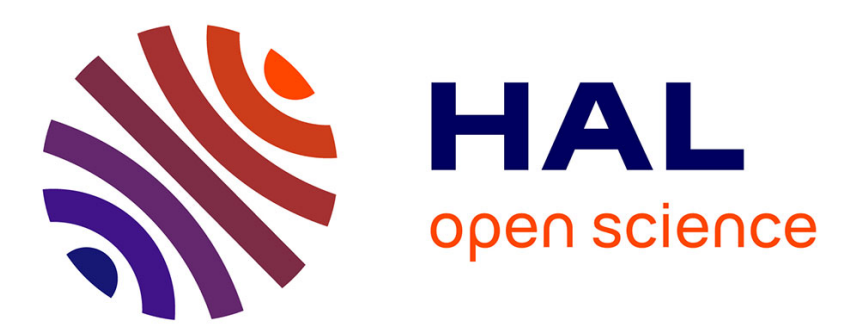

\title{
Nouvelles approches thérapeutiques de l'inflammation et de la progression dans la sclérose en plaques (évaluations cliniques et IRM)
}

P. Vermersch

\section{- To cite this version:}

P. Vermersch. Nouvelles approches thérapeutiques de l'inflammation et de la progression dans la sclérose en plaques (évaluations cliniques et IRM). Pratique Neurologique - FMC, 2019, 10, pp.126 130. 10.1016/j.praneu.2019.02.008 . hal-03486554

\section{HAL Id: hal-03486554 \\ https://hal.science/hal-03486554}

Submitted on 21 Dec 2021

HAL is a multi-disciplinary open access archive for the deposit and dissemination of scientific research documents, whether they are published or not. The documents may come from teaching and research institutions in France or abroad, or from public or private research centers.
L'archive ouverte pluridisciplinaire HAL, est destinée au dépôt et à la diffusion de documents scientifiques de niveau recherche, publiés ou non, émanant des établissements d'enseignement et de recherche français ou étrangers, des laboratoires publics ou privés.

\section{(ㄷ)(1) $\$$}

Distributed under a Creative Commons Attribution - NonCommerciall 4.0 International 


\section{Nouvelles approches thérapeutiques de l'inflammation et de la progression} dans la sclérose en plaques (évaluations cliniques et IRM)

New therapeutic strategies of inflammation and progression in multiple sclerosis (Clinical and imaging evaluation)

Patrick Vermersch

Univ. Lille, CHU Lille, LIRIC - INSERM U995, FHU Imminent, F-59000 Lille

Adresse de correspondance: Patrick Vermersch, Clinique de Neurologie, Hôpital Roger Salengro, CHU de Lille, Avenue Emile Laine, 59037 Lille cedex, France

Mél : patrick.vermersch@univ-lille.fr

Tél : (33)-3-20445765

Fax : (33)-3-20444484 


\title{
Résumé
}

La physiopathologie de la sclérose en plaques est encore partiellement inconnue, limitant le nombre de nouvelles approches thérapeutiques. Le traitement repose encore presque exclusivement sur le contrôle des paramètres inflammatoires. A côté de l'approche conventionnelle basée sur l'escalade thérapeutique, les thérapeutiques de reconstitution immunitaire, basées sur une phase de déplétion suivie d'une phase de renouvellement des cellules immunitaires ayant un profil plus tolérogène, sont une alternative intéressante notamment dans les formes rémittentes très actives. Les traitements ciblant plus spécifiquement les lymphocytes B sont déjà aussi davantage prescrits, y compris dans les formes progressives avec une activité surajoutée, clinique ou même simplement radiologique. Même si à ce jour, la prise en charge des formes progressives est décevante, de nouvelles pistes thérapeutiques semblent prometteuses.

Mots-clefs: Sclérose en plaques, approches thérapeutiques, nouveaux traitements, anticorps monoclonaux

\begin{abstract}
The pathophysiology of multiple sclerosis remains partially unknown, limiting the number of new therapeutic approaches. The treatment is almost exclusively based on the control of the inflammatory component. Besides the most conventional approach based on escalation maintenance therapy, immune reconstitution therapies are no more frequently considered in the decision, mainly in highly active patients. After a depletive phase, the reconstitution period shows a more tolerogenic profile of the immune cells. Therapeutic agents targeting the $\mathrm{B}$ cells are also more considered in relapsing but also in progressive patients, in case of superimposed clinical or imaging activities. Even today disappointing, new therapeutic strategies have emerged to treat progressive patients with promising preliminary results.
\end{abstract}

Key words: Multiple sclerosis, therapeutic approaches, new drugs, monoclonal antibodies 


\section{Introduction}

Ces dernières années, la thérapeutique de la sclérose en plaques (SEP) a beaucoup évolué, notamment dans les formes rémittentes. Toutefois les décisions thérapeutiques restent difficiles. S'il est unanimement admis qu'un traitement de fond doit être instauré dès que possible dans les formes rémittentes, la décision d'un changement de traitement ne répond pas à un schéma consensuel. Les approches nouvelles évaluent mieux le rapport bénéfice risque des traitements et une tendance se dessine à l'utilisation précoce de thérapeutiques plus efficaces. Enfin même si ceux-ci ont un impact encore modeste, les résultats dans les formes progressives sont plus encourageants.

\section{Eléments physiopathologiques de l'inflammation et de la progression}

Dorénavant la classification des phénotypes de SEP considère plus distinctement ce qui est en rapport avec l'activité et ce qui est en rapport avec la progression (1). En règle, on considère l'activité définie soit cliniquement par une poussée soit radiologiquement par l'apparition de nouvelles lésions en modalités T2 ou en T1 rehaussées par le gadolinium comme étant la conséquence d'une activité inflammatoire focale. Ce processus sous-tendant cette inflammatoire focale fait largement intervenir à la fois les lymphocytes $\mathrm{T}$ et $\mathrm{B}$, des dépôts d'immunoglobulines et de complément, directement ou indirectement responsable de la démyélinisation et de la souffrance axonale. La progression fait référence à l'évolution du handicap neurologique en règle assimilé à l'invalidité, en général mesuré par l'Expanded Disability Status Scale (EDSS) ou par des mesures plus écologiques comme le périmètre de marche, la vitesse de marche, l'agilité des membres supérieurs ou encore par des tests cognitifs. Les bases fondamentales de la progression sont moins bien élucidées. Sur le plan anatomique, la progression de la maladie mesurée par le handicap est davantage corrélée aux lésions diffuses en substance blanche, aux lésions corticales et de façon plus générale aux mesures d'atrophie, qu'aux lésions focales, même si celles-ci y contribuent. Sur le plan 
cellulaire, si les lymphocytes jouent encore un rôle, d'autres éléments constitués interviennent notamment les formes dites progressives notamment les cellules microgliales et les astrocytes. De même, un rôle important semble dévolu aux follicules lymphoïdes notamment dans la progression. Ces follicules localisés dans les méninges et les zones sous-piales sont particulièrement riches en cellules de la lignée lymphocytaire B et contribuent certainement à la démyélinisation et aux lésions axonales observées dans le cortex (2). Les mécanismes de la dégénérescence neuronale sont divers mais incertains. Ils pourraient varier d'un individu à l'autre et être différents chez un même individu au cours des différentes phases de la maladie. Il est clair que la progression de la maladie est corrélée à l'importance de ces lésions dégénératives. La démyélinisation est un élément majeur de susceptibilité à la dégénérescence. Sans le rôle mécanique de la myéline, l'axone sera fragilisé par les modifications du milieu comme la température, les modifications ioniques, ou de $\mathrm{pH}$. La démyélinisation entraine également une redistribution des canaux ioniques à la surface de l'axone afin de préserver une conduction nerveuse non saltatoire mais qui conduit plus ou moins rapidement à une accumulation intracellulaire de calcium, délétère pour l'axone. D’autres mécanismes contribuent aux lésions dégénératives notamment lié à la toxicité du glutamate ou liés à des altérations du métabolisme mitochondrial (pour revue 3).

\section{Nouvelles approches thérapeutiques de l'inflammation et de la progression}

Une des nouvelles façons d'appréhender la stratégie thérapeutique dans les formes rémittentes est de séparer l'approche conventionnelle du type escalade thérapeutique d'une approche plutôt d'induction. En règle l'escalade considère dans un premier temps l'utilisation d'un traitement dit de $1^{\text {ère }}$ ligne soit un immunomodulateur notamment les produits injectables (interférons béta ou acétate de glatiramère) ou le diméthyl-fumarate, soit un immunosuppresseur comme le tériflunomide. Si ces produits ne sont pas globalement considérés comme les plus actifs, de nombreux patients seront des répondeurs et ils 
bénéficient d'un bon rapport bénéfice risque. Il n'y a pas de consensus pour définir l'échec mais les algorithmes de suivi prennent en compte l'évolution clinique et IRM sous traitement, considérant aussi le délai d'action du médicament, et il faut donc idéalement avoir une IRM de référence après quelques mois de traitement. En cas de réponse jugé insuffisante, l'escalade se fait vers des thérapeutiques immunosuppressives comme le natalizumab, le fingolimod ou encore l'ocrelizumab. La décision de prescrire le natalizumab ou de l'arrêter repose surtout la stratification du risque de leuco-encéphalopathie multifocale progressive basé sur l'index du virus JC. Ces traitements peuvent être utilisés d'emblée dans des formes très actives ou même simplement jugés actives pour l'ocrelizumab. Outre les risques de l'immunosuppression continue avec ces produits, il existe un risque significatif de reprise d'activité de la maladie à l'arrêt voir de rebond sévère.

La démonstration du rôle important des lymphocytes B dans la physiopathologie de la SEP que ce soit dans les formes rémittentes mais aussi dans les formes progressives primaires, les résultats acquis (4) et le schéma simple de prescription feront des thérapeutiques ciblant les lymphocytes B par des anticorps anti-CD20 une approche commune. Néanmoins, même si la tolérance est satisfaisante après 5 ans d'utilisation d'ocrelizumab (5), il existe un léger risque infectieux, notamment broncho-pulmonaire, incitant une rigueur dans le suivi et une mise à jour du calendrier vaccinal, et une forte incitation à la vaccination anti-pneumococcique. D'autres molécules ciblant le CD20, comme l'ublitixumab sont en cours d'évaluation. Une piste nouvelle est en cours d'études pour combattre cette activité lymphocytaire B, notamment leur prolifération et leur capacité à produire des immunoglobulines et des cytokines, en agissant sur un enzyme, la Bruton tyrsosine kinase (BTK). Ces inhibiteurs de BTK (BTKi) pourraient aussi inhiber les macrophages. Cette dualité d'action semble donc très prometteuse pour les formes rémittentes mais potentiellement aussi dans les formes 
progressives. L'évobrutinib est le premier BTKi à avoir terminé une phase II dans la forme rémittente, avec des résultats positifs (6).

Une nouvelle approche, appelée traitement de reconstitution immunitaire (IRT) consiste à induire des modifications plus durables du système immunitaire, au-delà de la phase thérapeutique (7)(Figure 1). L'objectif est, après une phase de déplétion parfois profonde, limitée ou non aux lymphocytes, de faire émerger durant la phase de reconstitution immunitaire, un profil de lymphocytes plus tolérogènes. Cette IRT peut-être non spécifique avec la mitoxantrone ou l'autogreffe de moelle osseuse avec des modifications immunologiques significatives au-delà des lymphocytes, ou dite spécifique avec des changements importants mais limités essentiellement aux lymphocytes B et T. Même si la procédure est peu pratiquée car associée à des risques infectieux importants, l'autogreffe de moelle osseuse est le modèle de cette stratégie d'IRT (8). En effet le pourcentage de patients mis en rémission est élevé et de façon parfois très prolongée. Également, même si l'accès à ce traitement est limité, l'alemtuzumab cible le CD52, entraine une déplétion importante puis une phase de reconstitution immunitaire prolongée avec des changements significatifs du profil immunitaire pouvant persister parfois plusieurs années après le dernier cycle de perfusion, notamment pour les lymphocytes T CD4 (9). Dans les études évaluant l'alemtuzumab, entre 50 à $60 \%$ de patients sont restés sans traitement jusqu'à 6 ans après la fin de la phase thérapeutique. La cinétique de reconstitution immunitaire très différente et plus rapide pour les lymphocytes $\mathrm{B}$ que pour les lymphocytes $\mathrm{T}$, explique probablement le risque élevé de pathologies dysimmunitaires secondaires, notamment thyroïdiennes ou encore des thrombopénies auto-immunes parfois plusieurs années après les cures d'alemtuzumab, obligeant à une surveillance biologique rapprochée et durable (10). La cladribine avec seulement 20 jours de traitement par voie orale réparti sur 2 ans, entraine également un 
contrôle durable documenté chez près de $75 \%$ des patients durant environ 4 ans, soit près de 3 ans après la dernière prise de cladribine (11).

\section{Les nouvelles approches de la progression}

Les nouveautés dans les formes progressives obtenues durant les 5 dernières années sont malheureusement peu nombreuses. Les meilleurs résultats obtenus dans les formes récurrentes rémittentes laissent espérer un risque plus faible et retardé de passage à la forme secondairement progressive. Les analyses des patients traités par des thérapeutiques très actives comme le natalizumab ou l'alemtuzumab lors de la phase rémittente sont très encourageantes. Les données obtenues avec l'ocrelizumab dans l'étude Oratorio ont montré pour la première fois, dans une population de patients uniquement atteints de formes progressives primaires, une diminution du risque de progression confirmée de leur invalidité mesurée par l'EDSS (12). Les objectifs secondaires étaient également atteints, sur le plan clinique avec la vitesse de marche au test des 25 pieds ou de fonctions des membres supérieurs au test des 9 trous, ou en imagerie sur les paramètres d'atrophie. En ciblant le CD20, outre la déplétion au niveau périphérique, il est évoqué la possibilité d'atteindre les lymphocytes B présents en nombre dans les espaces méningés, notamment dans les follicules lymphoïdes. D'autres thérapeutiques ciblant les cellules immunitaires et ayant une bonne pénétration dans le SNC, comme la cladribine, sont prometteuses.

Le challenge des thérapeutiques de la progression se situe néanmoins dans les possibilités de remyélinisation et de neuroprotection. Ces deux mécanismes sont très liés, la perte axonale étant très favorisée par la démyélinisation. Un faible pourcentage de patients a été amélioré par de fortes de doses de biotine (13), permettant d'obtenir une autorisation temporaire d'utilisation dans les formes progressives. La biotine à dose élevée pourrait compenser le déficit énergétique des neurones et stimuler la remyélinisation. Il est difficile de dire s'il s'agit d'un traitement symptomatique ou modifiant l'évolution de la maladie. La mise sur le marché 
dépendra des résultats d'une autre étude en cours aux USA. Même si les premiers résultats évaluant l'opicinumab sont un peu décevants, les recherches se poursuivent car les études ont objectivé des résultats positifs sur des paramètres neuro-visuelles ou encore d'imagerie (14). Dans différents modèles cellulaires ou animaux, l'opicinumab favorise la remyélinisation en inhibant le lingo 1, molécule bloquant physiologiquement les capacités de réparation. Une équipe de SanFrancisco, utilisant un système de screening original et à grande échelle, ont sélectionné un antihistaminique utilisé aux USA, la clémastine, comme agent favorisant la différenciation des oligodendrocytes (15). Dans une étude clinique préliminaire avec cross over incluant 50 patients avec séquelles stables de neuropathies optiques, les auteurs ont observés sous clémastine par rapport au placebo une amélioration significative de la latence des potentiels évoqués et une discrète amélioration d'un test de reconnaissance des lettres (16). Une piste très originale a été présentée avec là aussi des résultats préliminaires encourageants pour la progression. En effet ce traitement évalué au cours d'une phase II ne semble pas avoir d'effets sur les paramètres inflammatoires cliniques ou d'imagerie mais par contre diminue très significativement le taux d'atrophie (17). Ce produit relance par ailleurs le débat sur le rôle potentiel des séquences rétrovirales dans la SEP. En effet il s'agit d'un anticorps monoclonal (GNbAC1) qui inhibe la pathogénicité d'un rétrovirus endogène (HERV-W) en diminuant notamment l'activité des cellules microgliales. Des données expérimentales montrent que cet anticorps pourrait avoir une activité pro-myélinisante. Une autre étude a confirmé aussi l'intérêt de l'Ibudilast dans les formes progressives. Cette petite molécule dont le mécanisme d'action est peu connu, pénètre bien le SNC et aurait une action neuroprotectrice. Les résultats ont montré là aussi une diminution nette des paramètres d'imagerie associés à la progression par rapport au placébo comme l'atrophie globale, et de façon plus franche encore l'atrophie corticale ou le ratio de transfert de magnétisation (18). D'autres pistes n'ont pas confirmé les espoirs portés en eux comme l'étude MS-SMART, 
avec aucune différence en ce qui concerne le taux d'atrophie de la fluoxétine, du riluzole et de l'amiloride comparé au placebo chez des patients en formes progressives (19). La recherche se poursuit en ce qui concerne la voie des modulateurs des sphingosines-phosphates. En effet certains de ces modulateurs agissent davantage sur le sous-type 5 de ces récepteurs, exprimés sur les oligodendrocytes avec donc un potentiel de remyelinisation.

\section{Conclusion}

Les nouvelles approches thérapeutiques dans la SEP sont nombreuses. Il reste difficile en l'absence de biomarqueur fiable du pronostic d'établir un pronostic et donc de choisir les meilleures options. Néanmoins, même si certains traitements sont difficilement accessibles en France, les traitements de reconstitution immunitaire offrent une alternative très intéressante au schéma plus classique de l'escalade thérapeutique dans les formes sévères ou chez les patients cumulant des facteurs de mauvais pronostic. Le développement récent de l'approche ciblant les lymphocytes B a changé également notre vision de l'approche thérapeutique, notamment dans les formes progressives avec activité surajoutée. Quant aux nouvelles thérapeutiques, si les analyses permettent d'être optimiste dans les formes récurrentesrémittentes, les données cliniques dans les formes progressives restent beaucoup plus décevantes.

\section{Liens d'intérêts}

Honoraires de consultants, aide déplacement en congrès de Biogen, Merck, Sanofi, Novartis, Teva, Roche, Servier, Celgène, Medday.

Soutiens à la recherche de Biogen, Sanofi, Roche. 


\section{Points essentiels}

- Deux approches thérapeutiques peuvent être considérés dans les formes rémittentes : I'une traditionnelle avec escalade thérapeutique, l'autre basée sur des traitements de reconstitution immunitaire.

- La démonstration du rôle des lymphocytes $B$ et les résultats des études incitent à une prescription des biothérapies ciblant le CD20, y compris dans les formes progressives, lorsqu'une activité de la maladie est objectivée.

- Même si les résultats préliminaires sont souvent décevants, de nouvelles approches se développent dans les formes progressives, basées sur leur potentiel de remyélinisation et de neuroprotection. 


\section{Références}

1. Lublin FD, Reingold SC, Cohen JA, Cutter GR, Sørensen PS, Thompson AJ, et al. Defining the clinical course of multiple sclerosis: the 2013 revisions. Neurology 2014, 83: 278-286.

2. Magliozzi R, Howell O, Vora A, Serafini B, Nicholas R, Puopolo M, et al. Meningeal $\mathrm{B}$-cell follicles in secondary progressive multiple sclerosis associate with early onset of disease and severe cortical pathology. Brain 2017, 130: 1089-1104.

3. Lassmann H, Brück W, Lucchinetti CF. The Immunopathology of Multiple Sclerosis: An Overview. Brain Pathol 2007, 17, 210-218.

4. Hauser SL, BarOr A, Comi G, et al. Ocrelizumab versus interferon beta1a in relapsing multiple sclerosis. N Engl J Med 2017, 376: 22134.

5. Hauser S. Safety of ocrelizumab in multiple sclerosis: updated analysis in patients with relapsing and primary progressive multiple sclerosis. ECTRIMS 2018, Berlin. P1229

6. Montalban X. Primary analysis of a randomised, placebo-controlled, phase 2 study of the Bruton's tyrosine kinase inhibitor evobrutinib (M2951) in patients with relapsing multiple sclerosis. ECTRIMS 2018, Berlin. O322.

7. Giovannoni G. https://www.excemed.org/resources/how-transfer-concept-clinicalpractice [Accessed July 2017].

8. Mancardi G, Saccardi R. Autologous haematopoietic stem-cell transplantation in multiple sclerosis. Lancet Neurol 2008; 7: 626-36.

9. Baker D, Herrod SS, Alvarez-Gonzalez C, Giovannoni G, Schmierer K. Interpreting lymphocyte reconstitution data from the pivotal phase 3 trials of alemtuzumab. JAMA Neurol 2017c; 74: 961-9.

10. Baker D, Herrod SS, Alvarez-Gonzalez C, Zalewski L, Albor C, Schmierer K. Both cladribine and alemtuzumab may effect MS via B-cell depletion. Neurol Neuroimmunol Neuroinflamm 2017;4: e360; doi: 10.1212/NXI.0000000000000360

11. Giovannoni G, Comi G, Cook S, Rieckmann P, Rammohan K, Soelberg-Sorensen P, et al. Durable efficacy of cladribine tablets in patients with multiple sclerosis: analysis of relapse rates and relapse-free patients studies in the CLARITY and CLARITY EXTENSION studies. CMSC 2017, PDX30.

12. Montalban X, Hauser SL, Kappos L, Arnold DL, BarOr A, Comi G, et al. Ocrelizumab versus Placebo in Primary Progressive Multiple Sclerosis. New Engl J Med $2017 ; 376: 209-20$.

13. Tourbah A, Lebrun-Frenay C, Edan G, Clanet M, Papeix C, Vukusic S, et al. MD1003 (high-dose biotin) for the treatment of progressive multiple sclerosis: A randomised, double-blind, placebo-controlled study. Multiple Sclerosis J 2016, Vol. 22(13) 17191731.

14. Cadavid D, Balcer LJ, Galetta SL, et al. Axonal Protective Role of LINGO-1 Blockade in the Visual System: Results From a Study in Rats and Designof of a Phase 2 Clinical Trial for BIIB033, an Anti-LINGO-1 Monoclonal Antibody, in Subjects With a First Episode of Acute Optic Neuritis, Presented at: 66th Annual Meeting of the American Academy of Neurology, 26 April-3 May, Philadelphia, PA, USA, 2014.

15. Mei F, Fancy SPJ, Shen YU, Niu J, Zhao C, Presley B, et al. Micropillar arrays as a high-throughput screening platform for therapeutics in multiple sclerosis. Nat Med 2014; 20: 954-961. 
16. Green A. Promoting endogenous remyelination: from basic sciences to translation. ECTRIMS 2018, Berlin. O116.

17. Hartung HP. Week 48 results from a phase IIb trial of GNbAC1 in patients with relapsing remitting multiple sclerosis (CHANGE-MS; clinical trial assessing the HERV-W Env antagonist GNbAC1 for Efficacy in MS). ECTRIMS 2018, Berlin. O143.

18. Fox RJ, Coffey CS, Conwit R, Cudkowicz ME, Gleason T, Goodman A, et al. Phase 2 Trial of Ibudilast in Progressive Multiple Sclerosis. N Engl J Med. 2018 Aug 30; 379: 846-855.

19. Chataway J. MS-SMART Trial: a multi-arm phase $2 \mathrm{~b}$ randomised, double blind, parallel group, placebo-controlled clinical trial comparing the efficacy of three neuroprotective drugs in secondary progressive multiple sclerosis [NCT01910259] . ECTRIMS 2018, Berlin. O324. 
Figure 1: Classification des approches thérapeutiques dans les formes rémittentes de SEP (d'après 7 modifiée) : TMO : transplantation de moelle osseuse 


\section{Une nouvelle classification des traitements de fond dans la SEP-R}

\section{Thérapies de Maintenance (MT)}

- Traitements chroniques maintenus dans le temps pour observer une efficacité durable : administration continue

- L'effet thérapeutique sur le système immunitaire est présent uniquement durant la période de traitement et disparaît à l'arrêt de celui-ci
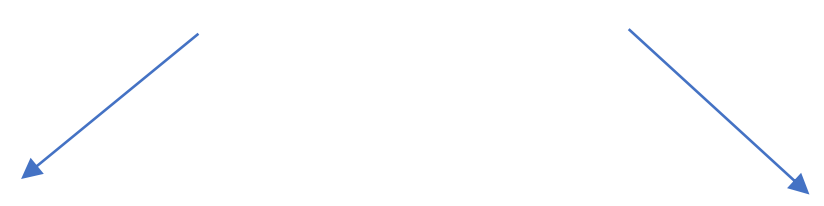

\section{Immunomodulation . . . . Immunosuppression}

Thérapie de Maintenance (MET) qui entraîne une immunomodulation continue

IFNbeta, Acetate de Glatiramère
Thérapie de Maintenance (MET) qui entraîne une immunosuppression continue

Natalizumab Fingolimod, Ocrelizumab

\section{Thérapies de Reconstitution Immunitaire (IRT)}

- Traitements séquentiels par courtes périodes : administration séquentielle

- Le traitement remodèle le système immunitaire : cela entraîne des changements qualitatifs dans la fonction immunitaire et permet une efficacité durable sans retraitement systématique nécessaire

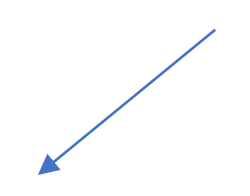

IRT Sélective (SIRT)

Thérapie de Reconstitution Immunitaire (IRT) avec une action sélective sur le système immunitaire adaptatif, préservant ainsi en grande partie l'immunité innée Alemtuzumab, Cladribine

\section{IRT Non Sélective} (NIRT)

Thérapie de Reconstitution Immunitaire (IRT) avec une action non sélective, qui impacte à la fois le système immunitaire inné et adaptatif

Mitoxantrone, TMO 